\title{
Identification and Functional Validation of a Biomarker for the Diagnosis of Miltefosine Relapse during Visceral Leishmaniasis
}

\author{
Puja Tiwary, Dinesh Kumar, and Shyam Sundar* \\ Department of Medicine, Infectious Disease Research Laboratory, Institute of Medical Sciences, Banaras Hindu University, \\ Varanasi, Uttar Pradesh, India
}

\begin{abstract}
Miltefosine is the only orally administrable drug for the treatment of leishmaniasis. But in recent years, a decline in its efficacy points toward the emergence of resistance to this drug. Knowledge of biomarkers for miltefosine resistance may be beneficial for proper selection of treatment regimen. Splenic aspirates were collected and parasites cultured from patients relapsed after initial cure $(N=15)$ and successfully treated $(N=15)$ with miltefosine. Differential expression of genes in miltefosine-resistant strains was examined by DNA microarray and validated by real-time reverse transcription polymerase chain reaction and Western blotting. Of 669 upregulated genes, the cysteine protease-like protein of calpain family (GenBank: CBZ34784) was found to be significantly overexpressed in resistant parasite strains and only anti-calpain antibodies showed its presence in the sera of relapse patients through Western blotting. Calpain family cysteine protease-like protein can be useful as a potential biomarker of miltefosine unresponsiveness.
\end{abstract}

\section{INTRODUCTION}

Visceral leishmaniasis (VL) is the most severe form of leishmaniasis and is fatal if untreated. Unfortunately, the inventory of antileishmanial drugs is very small, consisting of sodium antimony gluconate (SAG), miltefosine, paromomycin, and amphotericin B. Pentavalent antimonials remained effective until 1980s when in India and to some extent in Nepal, failure of SAG treatment was reported. ${ }^{1}$ Oral miltefosine, for its ease of use in program conditions, was selected as the treatment in the VL Elimination Program launched by India, Nepal, and Bangladesh in $2005 .^{2}$ Because of the long half-life of miltefosine (MIL), it is more prone to the development of resistance. ${ }^{3}$ A study conducted a decade after its pivotal phase 3 trials showed more than doubling of relapses from $3 \%$ to $7.24 \%$ after 6 months. ${ }^{4}$ Because miltefosine is used on such a wide scale in the Elimination Program, identification of biomarkers of miltefosine resistance will be helpful in selecting patients less likely to respond to it.

Leishmania donovani promastigote in vitro selected for MIL resistance showed changes in the length and level of unsaturation of fatty acids, a reduction in ergosterol levels, and more than $95 \%$ reduction in drug internalization which was consistently associated with mutations in the MIL transporter genes LdMT and LdRos3. ${ }^{5}$ Considering that the reasons may be different in actual clinical resistant strains, we designed a study to identify biomarkers of miltefosine resistance in patients with VL.

\section{MATERIALS AND METHODS}

Study area. This study was conducted at the Infectious Diseases Research Laboratory, Department of Medicine, Institute of Medical Sciences, Banaras Hindu University, and its field site at the Kala-azar Medical Research Center, Muzaffarpur, Bihar. Ethical approval was obtained from the Ethics Committee of the Institute of Medical Sciences, Banaras Hindu University. Written informed consent was obtained from all patients or their guardians.

*Address correspondence to Shyam Sundar, Department of Medicine, Institute of Medical Sciences, Banaras Hindu University, Varanasi221005, Uttar Pradesh, India. E-mail: drshyamsundar@hotmail.com
Collection of parasite strains. After successful treatment with MIL, we followed up patients for 6 months. We found some patients with relapse of VL. We recruited such patients again for treatment and splenic aspirates were collected as relapse sample, whereas control samples were splenic aspirates collected from patients cured after miltefosine treatment and did not relapse. Aspirates microscopically confirmed for Leishmania parasites were added to Novy-McNeal-Nicolle tubes, supplied M199 medium, and kept in the incubator at $26^{\circ} \mathrm{C}$. The cultures were examined weekly for parasite growth and secondary culture was carried out in culture flasks with M199 media.

Serum collection. Two milliliter of venous blood from patients and healthy controls was collected. Blood was centrifuged at 4,000 rpm for 2 minutes to separate serum, which was stored at $-20^{\circ} \mathrm{C}$.

RNA extraction. The parasite culture was pelleted by centrifugation at 5,000 rpm for 10 minutes, washed twice with $1 \times$ phosphate-buffered saline (PBS), and mixed with RNAlater (Qiagen, Hilden, Germany) for storage at $-80^{\circ} \mathrm{C}$ until RNA extraction. RNA was isolated using the RNeasy mini kit (Qiagen) as per the manufacturer's protocol and eluted in $30 \mu \mathrm{L}$ RNase free water (Qiagen). RNA of each sample was quantified using Nano Drop 2000c and quality-checked based on the ratio of A260/A280, which was found to be in the range of 1.9-2.0 for all the samples. Total RNA integrity was evaluated using an RNA 6000 Nano Assay kit on Bioanalyzer 2100 (Agilent Technologies, Santa Clara, CA), and RIN (RNA integrity number) values for all the samples were satisfactory and above 7 .

DNA microarray and data analysis. The microarray experiment was performed by Genotypic technology (Bangalore, India) based on a one color experiment on an Agilent microarray platform. Leishmania donovani-specific biochip was custom designed by Agilent technology based on recently submitted whole-genome sequencing data for the same. ${ }^{6}$ We preferred a single color experiment, in which each sample was labeled with Cy3 and then hybridized in individual arrays. Biological replicates of each phenotype were hybridized separately in arrays. Each array was then normalized separately (Intra-array) and then against control (Inter-array). The advantage of a single color experiment is that many test samples can be compared with the control without hybridizing the same control with each sample every time. 
TABLE 1

Primer sequences

\begin{tabular}{|c|c|c|c|c|}
\hline Gene & Technique & Forward $\left(5^{\prime}-3^{\prime}\right)$ & Reverse $\left(5^{\prime}-3^{\prime}\right)$ & $\operatorname{Tm}\left({ }^{\circ} \mathrm{C}\right)$ \\
\hline Transporter-like protein & RT-PCR & GGGATCTGGTACGTCTATCTGT & GCTGCACGACAAGGGTAAA & 55 \\
\hline Hypothetical protein (1) & RT-PCR & CGCCATCAAGGGCATCTITA & CACCCGCTTATGTTCAGGTT & 55 \\
\hline Calpain family cysteine protease-like protein & RT-PCR & CGAAGCAACACCGATACTGA & CTACCGCATCTTCCGAGTTT & 55 \\
\hline Hypothetical protein (2) & RT-PCR & AACAAGGTCGGCCGTAAA & CAGAAGCACAACATCCACAAG & 55 \\
\hline Calpain family cysteine protease-like protein & Cloning & $\begin{array}{l}\text { GGATTCATGGGCAACGGTTGCT } \\
\text { GCGGCTGC }\end{array}$ & $\begin{array}{l}\text { GAATTCCATCACCAGCTCGAGC } \\
\text { TCCGCGTTGTG }\end{array}$ & 66 \\
\hline Hypothetical protein (2) & Cloning & $\begin{array}{l}\text { GAGCTCATGAGCCGCAGTCCCA } \\
\text { AGCAGCACCG }\end{array}$ & $\begin{array}{l}\text { GAATTCCTGATCGGCCTCTCTG } \\
\text { CGTCTCTCGTC }\end{array}$ & 67 \\
\hline
\end{tabular}

RT-PCR = reverse transcription polymerase chain reaction. Underlined sequence represents the restriction enzyme recognition sites.

Data analysis for DNA microarray. Extracted data were analyzed using GeneSpring Gx version 10.0.1. Normalization of the data was carried out using percentile shift normalization. Differentially regulated genes were clustered using gene tree to identify significant gene expression patterns. Ontologybased biological analysis was carried out using the Gene Ontology browser in GeneSpring Gx version 10.0.1. Furthermore, biological analysis was performed using Genotypic's Biointerpreter tool.

Database submission of microarray data. The data discussed in this publication have been deposited in the National Center for Biotechnology Information's Gene Expression Omnibus ${ }^{7}$ and are accessible through GEO Series accession number GSE53738 (http://www.ncbi.nlm.nih.gov/geo/query/ acc.cgi?acc $=$ GSE53738).

Quantitative real-time RT-PCR analysis. Real-time reverse transcription polymerase chain reaction (RT-PCR) was performed for the validation of microarray data on a larger sample size based on SYBR Green chemistry. More than 2.5-fold overexpressed genes were validated by real-time RT-PCR for the larger sample size. A volume of $1 \mu \mathrm{g}$ RNA isolated from all parasite strains was reverse transcribed in a $20-\mu \mathrm{L}$ reaction using a High Capacity cDNA Reverse Transcription kit (Invitrogen) and diluted 10 times with RNase-free water to a final concentration of $5 \mathrm{ng} / \mu \mathrm{l}$.

Primers specific for the 18S rRNA encoding gene of L. donovani were used as endogenous control. ${ }^{8}$ Forward and reverse primers for RT-PCR were designed for the targeted genes, expressing more than 2.5-fold in resistant than in sensitive samples (Table 1). One microliter cDNA of each sample was used in a $20-\mu \mathrm{L}$ reaction mixture with $5 \mathrm{pM}$ primers specific for targeted protein and endogenous control along with $10 \mu \mathrm{L}$ of $2 \times$ SYBR Green reagents (Applied Biosystems). The reactions were run using MicroAmp optical 96-well reaction plates (Applied Biosystems) and run on a 7500 Real-Time PCR System (Applied Biosystems). All samples were run in duplicate along with nil template master mix as negative controls in each plate.

Data analysis for real-time RT-PCR. For each sample, threshold cycle $\left(\mathrm{C}_{\mathrm{T}}\right)$ value was obtained directly by using 7500 software v2.0.1 (Applied Biosystems). Relative gene expression over the endogenous control gene was quantified using $\Delta \mathrm{C}_{\mathrm{T}}$ value. Differential gene expression of resistant parasites over sensitive ones was calculated as $\Delta \Delta \mathrm{C}_{\mathrm{T}}$. Then the normalized expression ratio was calculated as $2^{-\Delta \Delta C T}$. To identify significant overexpressed gene, the unpaired student $t$ test was applied with a $P$ value $<0.05$.

Cloning, expression, and purification. Differentially expressed genes, verified by both microarray and quantitative RT (qRT)-PCR, were further validated by Western blotting after producing recombinant proteins using the method described previously. ${ }^{10}$ Primers were designed for target proteins and annealing temperature (Table 1).

Immunoblotting. Purified recombinant proteins were run on $12 \%$ sodium dodecyl sulfate polyacrylamide gel electrophoresis and transferred to a PVDF (polyvinylidene difluoride) membrane $(0.45 \mu \mathrm{m}$ pore size, Millipore, USA) using a Bio-Rad trans-blot apparatus. The membrane was hybridized with primary antibodies, i.e., sera (1:100 in PBS) of four groups as mentioned earlier, for 1 hour at $37^{\circ} \mathrm{C}$. Alkaline phosphataseconjugated goat antihuman IgG $(1: 1,000)$ was used as a secondary antibody. Finally, color was developed using 5-bromo-4-chloro-3-indolyl phosphate-nitroblue tetrazolium as a substrate (Promega, USA).

\section{RESULTS}

For the present study, we collected splenic aspirates and sera from patients relapsing after the initial course of treatment $(N=15)$, confirmed by positive splenic score, and those cured with miltefosine treatment $(N=15)$ during 2011-2012.

DNA microarray. Differential gene expressions were calculated by transforming the intensity of expression into log2 ratio. A total of 7705 gene-specific probes were used to hybridize with cye3-labeled RNA of each sample. A histogram of

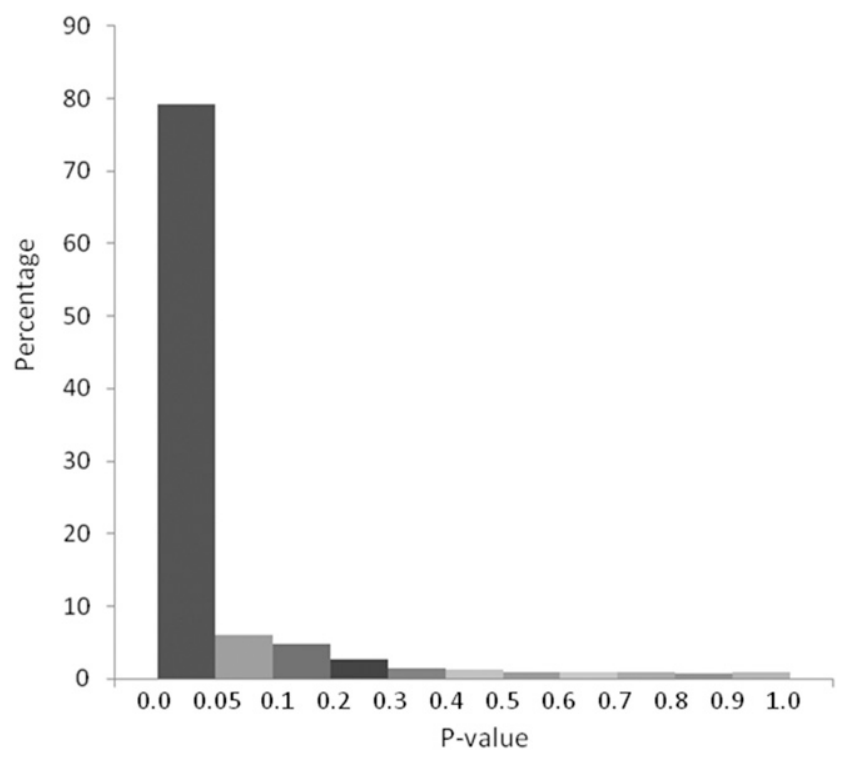

FIGURE 1. Percentage histogram of $P$ values of differentially expressed genes. 


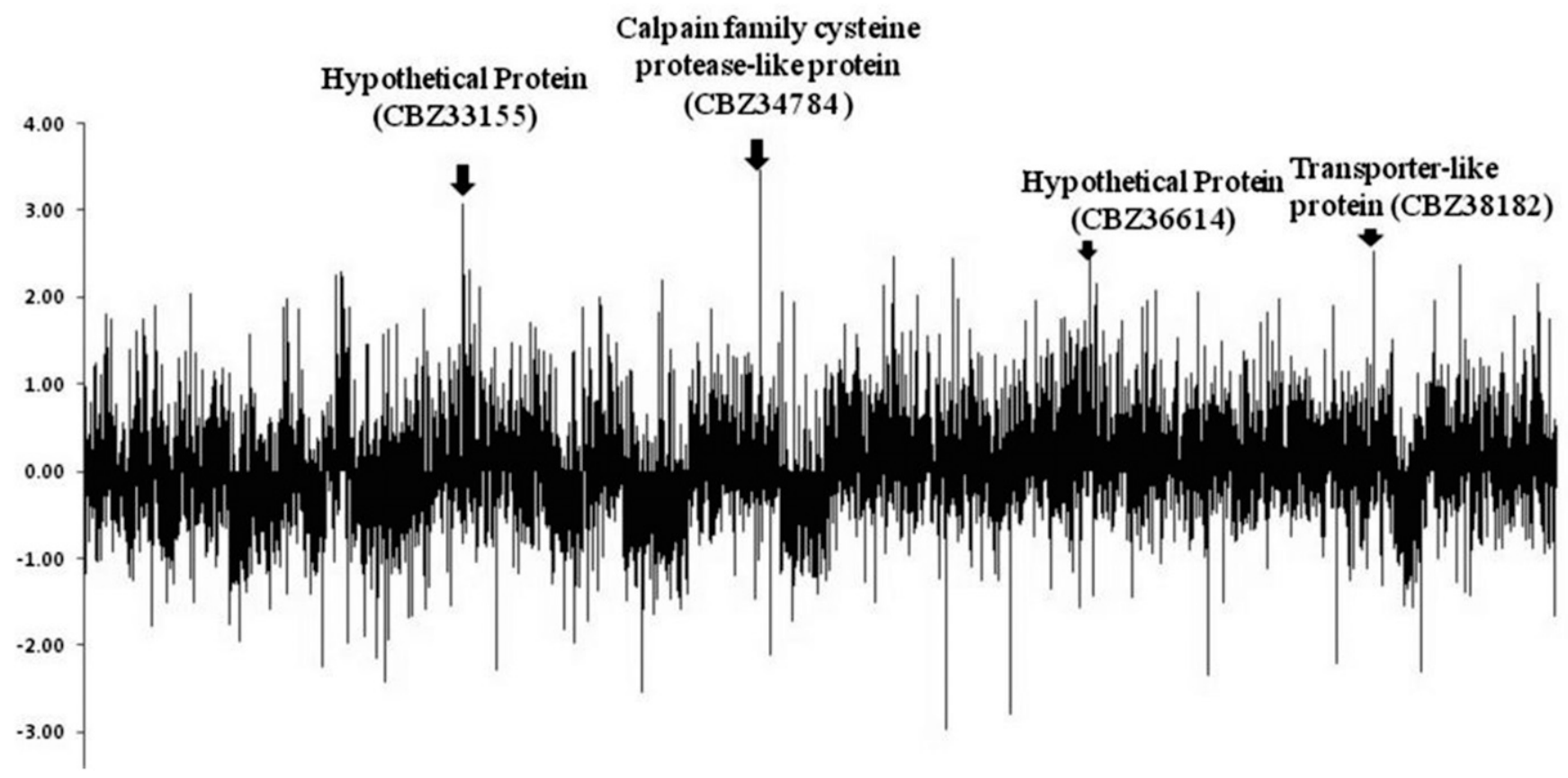

FIGURE 2. Microarray-based gene expression profile of miltefosine-relapsed parasites over sensitive strains. Vertical lines represent the log2transformed expression ratio of individual genes.

the $P$ value is shown in Figure 1. Six hundred sixty-nine genes were found to be overexpressed with a log2 ratio between 0.8 and 3.46. A total of 470 genes were found to be underexpressed with log2 ratio between -0.8 and -2.98 (Figure 2 and Table 2).

Quantitative real-time RT-PCR. A total of four proteins, with more than 2.5-fold overexpression in resistant samples, were selected for real-time assay and were identified as transporter-like proteins (GenBank: CBZ38182), hypothetical protein (GenBank: CBZ33155), calpain family cysteine protease-like protein (GenBank: CBZ34784), and another hypothetical protein (GenBank: CBZ36614). qRT-PCR was performed targeting these four proteins encoding genes for samples from 15 miltefosine-relapsed and 33 cured patients. The gene encoding for the putative calpain family cysteine protease-like protein and one hypothetical protein (GenBank: CBZ36614) were upregulated by 3.46- and 2.46-fold, respectively, using a DNA microarray. These were also found to be significantly upregulated by RT-PCR, with $P$ value $<0.0001$ and 0.0003 , respectively (Figure 3 ).

Cloning, expression, and purification of recombinant protein. Recombinant forms of calpain family cysteine protease-like protein and hypothetical proteins were successfully expressed and purified (Figure 4).

Immunoblotting. Only recombinant calpain protein was recognized by sera of resistant groups, whereas recombinant hypothetical protein was not recognized by sera of all the groups (Figure 4).

\section{DISCUSSION}

In the present study, we assessed the susceptibility/resistance of Leishmania parasite against miltefosine. Our objective was to find a suitable biomarker that can be used to easily identify miltefosine susceptibility/resistance. This study was significantly facilitated by various molecular techniques such as RNA extraction, microarray, real-time RT-PCR, and Western blotting.

Miltefosine is currently the latest and only oral antileishmanial that came out of drug discovery pipelines in the past few decades, but recent reports indicate a significant decline in its efficacy against VL in the Indian subcontinent. The high miltefosine relapse rate poses a major challenge for the current Kala-azar Elimination Program in the Indian subcontinent and other leishmaniasis control programs worldwide. Significantly lower cure rates, from Nepal ${ }^{11}$ and Bangladesh, ${ }^{12}$ have also been reported. This might be due to the development of a novel defense mechanism in parasites that works against the mode of action of miltefosine. Detecting such alterations might be beneficial in resolving resistance issues with drugs targeted against them.

Drug unresponsiveness arises because of parasite adaptations to their surroundings by evolving altered metabolic pathways. Gradually, these naturally selected strains lead to the emergence of drug-resistant or unresponsive parasites. Detection of biomarkers has become an attractive tool to monitor disease progression, in drug development programs, and in clinical trials. ${ }^{13} \mathrm{~A}$ biomarker might be any altered protein or gene that helps in preparing conditions suitable for parasite survival inside the host. Detection of biomarkers associated with the drug-resistant phenotype of $L$. donovani might be

TABLE 2

Results obtained from DNA microarray

\begin{tabular}{lccccc}
\hline Expression condition & $\begin{array}{c}\text { Total number } \\
\text { of genes }\end{array}$ & $\begin{array}{c}0.8 \text { - to } \\
\text { 1-fold }\end{array}$ & $\begin{array}{c}\text { 1- to } \\
\text { 2-fold }\end{array}$ & $\begin{array}{c}2 \text { - to } \\
\text { 3-fold }\end{array}$ & > 3-fold \\
\hline Upregulated & 669 & 266 & 380 & 21 & 2 \\
Down-regulated & 470 & 231 & 229 & 10 & 0 \\
\hline Number of genes in resistant strains found up- or downregulated in comparison to sensitive \\
strains.
\end{tabular}



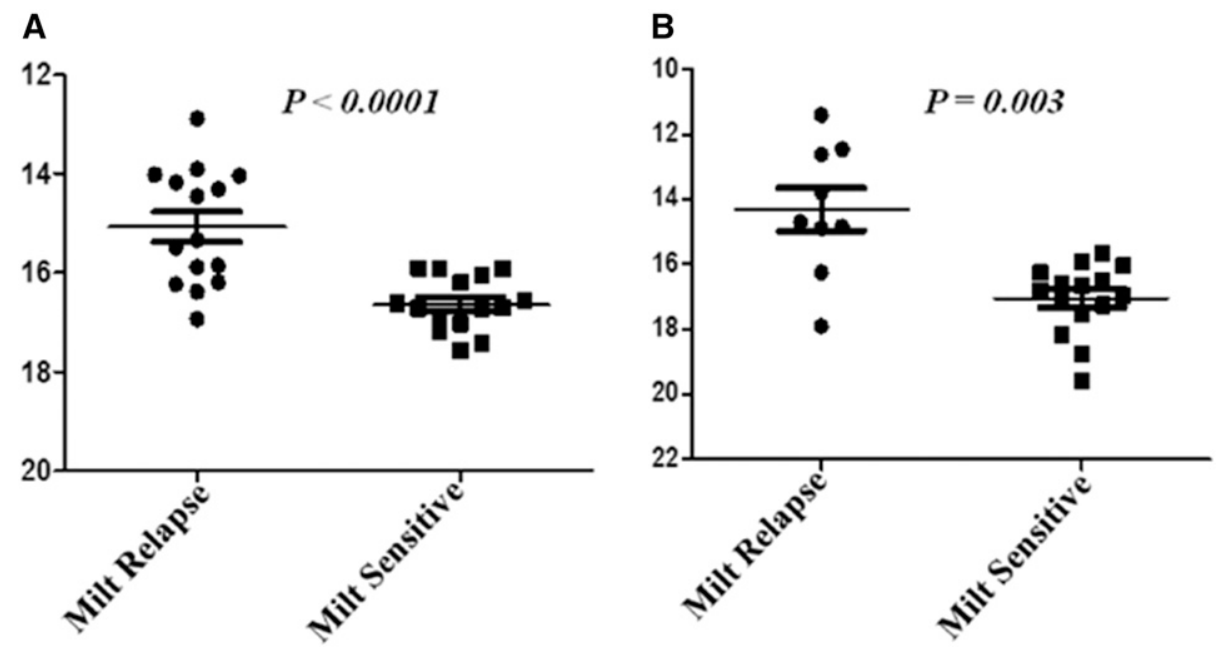

FIGURE 3. Differential gene expression of (A) calpain family cysteine protease-like protein and (B) hypothetical protein 2 using qualitative real-time reverse transcription polymerase chain reaction. Significant overexpression in miltefosine-relapsed parasites in comparison to sensitive strains.

helpful in early diagnosis of miltefosine unresponsiveness and in developing treatment regimens to cure patients effectively.

We collected clinically proven drug unresponsive parasites to observe the actual mechanism behind the resistance. Only calpain family cysteine protease-like protein was found to be significantly overexpressed in resistant parasites, and only anti-calpain antibody was significantly detected in the serum of miltefosine-relapsed patients. Calpain family cysteine protease-like protein (GenBank: CBZ34784) appeared to be a suitable candidate biomarker for miltefosine resistance as it qualified at each step of validation.
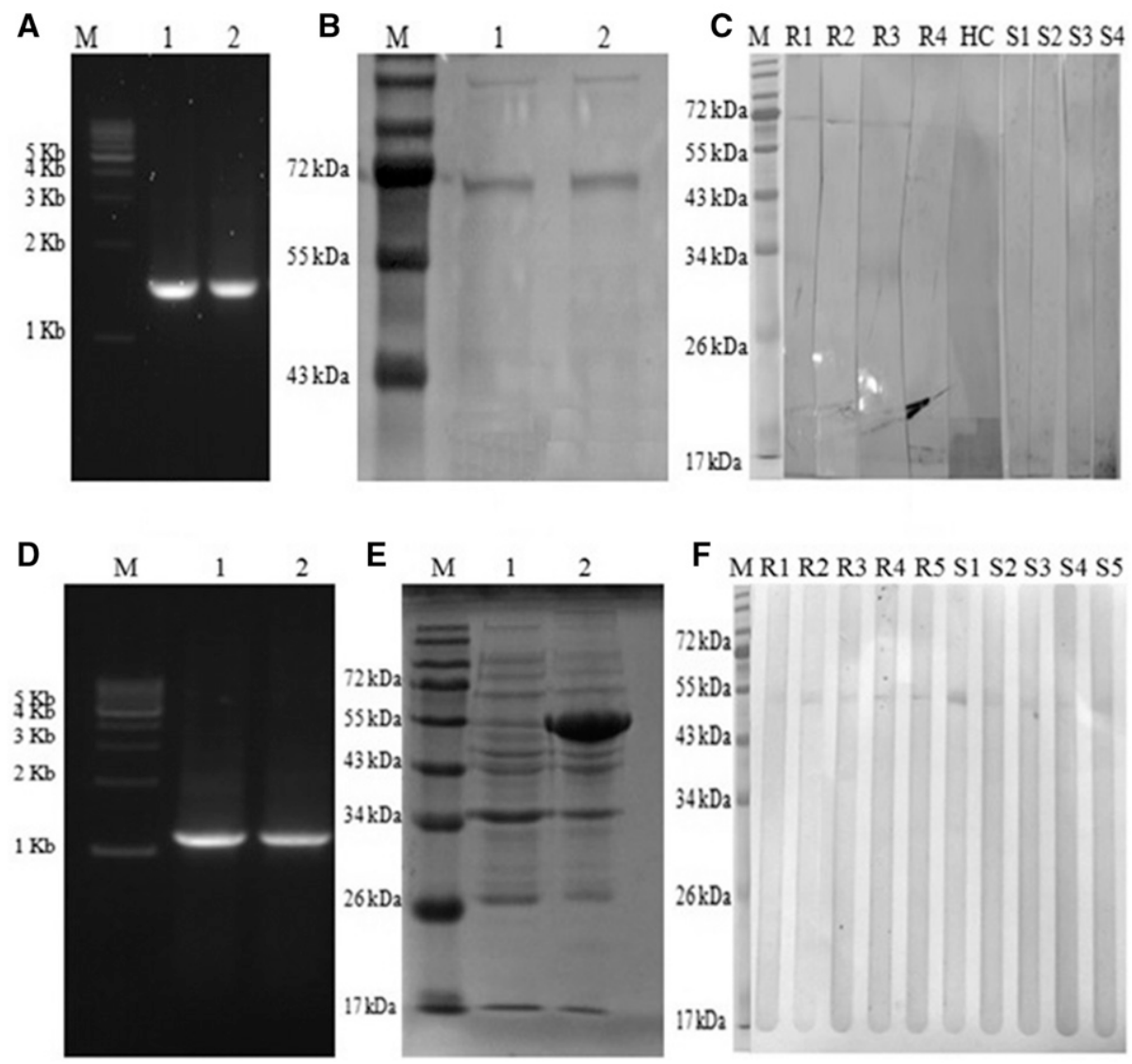

FIGURE 4. Cloning, expression, and immunoblotting of recombinant calpain family cysteine protease-like protein and hypothetical protein 2. (A and D) Polymerase chain reaction amplification of targeted gene. Lanes 1 and 2 with duplicate samples of amplified gene. (B and E) Expressed targeted proteins. Lanes 1 and 2 with duplicate samples of expressed protein. (C and $\mathbf{F}$ ) Western blotting of recombinant proteins against sera of three groups: miltefosine resistant ( $\mathrm{R} 1, \mathrm{R} 2, \mathrm{R} 3$, and $\mathrm{R} 4)$, miltefosine sensitive (S1, S2, S3, and S4), and healthy control (HC). 
Calpain is a class of cysteine protease family present in mammalian cells and is involved in various metabolic processes. It is known for its function in cytoskeletal protein rearrangement, signal transduction pathways, and apoptosis. These proteins have been shown to have a stable interaction with the cell membrane and might play a role in cellular remodeling; ${ }^{14}$ however, their function is not yet determined. Their involvement in host-parasite interaction ${ }^{15}$ suggests that these proteases may be useful for chemotherapeutic targets.

The present study showed an association of calpain family cysteine protease-like protein with miltefosine metabolism in Leishmania parasites, and its overexpression might act against the activity of the drug, thus promoting parasite survival in host macrophage. The actual mechanism of this interference needs to be explored in great detail with a lot of advance techniques. But, currently we have explored only its use as a diagnostic marker for the identification of miltefosine resistance in Indian population. As a biomarker, this protein may be beneficial to screen patients for miltefosine resistance before initiating any treatment.

Received December 15, 2016. Accepted for publication September 26, 2017.

\section{Published online December 26, 2017.}

Acknowledgments: We want to thank Genotypic Technology Pvt. Ltd., Bangalore, for providing us the microarray facility. The authors thank the hospital staff at the Kala-azar Medical Research Centre, Muzaffarpur, for their assistance in the collection of patient samples. We especially thank Dr. Rajiv Kumar and Neetu Singh for their support. The authors thank Dr. Phillip Lawyer for revising the manuscript for language and grammatical check.

Financial support: This research was funded by a grant from the $\mathrm{Na}-$ tional Institute of Allergy and Infectious Diseases, NIH, USA, vide grant no. P50AI074321. Puja Tiwary and Dinesh Kumar thank the Council of Scientific and Industrial Research (CSIR) for providing fellowships.

Authors' addresses: Puja Tiwary, Dinesh Kumar, and Shyam Sundar, Department of Medicine, Infectious Disease Research Laboratory, Institute of Medical Sciences, Banaras Hindu University, Varanasi-221005, Uttar Pradesh, India, E-mails: tiwarypuja@gmail.com, praja_dinesh@ yahoo.co.in, and drshyamsundar@hotmail.com.

\section{REFERENCES}

1. Sundar S, More DK, Singh MK, Singh VP, Sharma S, Makharia A, Kumar PC, Murray HW, 2000. Failure of pentavalent antimony in visceral leishmaniasis in India: report from the center of the Indian epidemic. Clin Infect Dis 31: 1104-1107.

2. WHO, 2005. Regional Strategic Framework for Elimination of Kalaazar from the South-East Asia Region (2005-2015). New Delhi: Regional Office for South-East Asia SEA-VBC-85 (Rev-1). Geneva, Switzerland: World Health Organization.

3. Sundar S, Olliaro PL, 2007. Miltefosine in the treatment of leishmaniasis: clinical evidence for informed clinical risk management. Ther Clin Risk Manag 3: 733-740.

4. Sundar S, Singh A, Rai M, Prajapati VK, Singh AK, Ostyn B, Boelaert M, Dujardin JC, Chakravarty J, 2012. Efficacy of miltefosine in the treatment of visceral leishmaniasis in India after a decade of use. Clin Infect Dis 55: 543-550.

5. Hefnawy A, Berg M, Dujardin JC, De Muylder G, 2016. Exploiting knowledge on Leishmania drug resistance to support the quest for new drugs. Trends Parasitol 33: 162-174.

6. Downing $T$ et al., 2011. Whole genome sequencing of multiple Leishmania donovani clinical isolates provides insights into population structure and mechanisms of drug resistance. Genome Res 21: 2143-2156.

7. Edgar R, Domrachev M, Lash AE, 2002. Gene expression omnibus: NCBI gene expression and hybridization array data repository. Nucleic Acids Res 30: 207-210.

8. Deborggraeve S, Boelaert M, Rijal S, De Doncker S, Dujardin JC, Herdewijn P, Buscher P, 2008. Diagnostic accuracy of a new Leishmania PCR for clinical visceral leishmaniasis in Nepa and its role in diagnosis of disease. Trop Med Int Health 13: 1378-1383.

9. Livak KJ, Schmittgen TD, 2001. Analysis of relative gene expression data using real-time quantitative PCR and the 2(-Delta Delta C(T)) method. Methods 25: 402-408.

10. Kumar D, Tiwary P, Dube A, Chakravarty J, Rai M, Sundar S, 2013. Cloning, expression and purification of $L$. donovani specific antigen for serodiagnosis of visceral leishmaniasis. $J$ Mol Biomark Diagn 4: 1000141.

11. Rijal S et al., 2013. Increasing failure of miltefosine in the treatment of kala-azar in Nepal and the potential role of parasite drug resistance, reinfection, or noncompliance. Clin Infect Dis 56: 1530-1538.

12. Rahman $\mathrm{M}$ et al., 2011. Phase IV trial of miltefosine in adults and children for treatment of visceral leishmaniasis (kala-azar) in Bangladesh. Am J Trop Med Hyg 85: 66-69.

13. Biomarkers Definitions Working Group, 2001. Biomarkers and surrogate endpoints: preferred definitions and conceptual framework. Clin Pharmacol Ther 69: 89-95.

14. Hertz-Fowler C, Ersfeld K, Gull K, 2001. CAP5.5, a life-cycleregulated, cytoskeleton-associated protein is a member of a novel family of calpain-related proteins in Trypanosoma brucei. Mol Biochem Parasitol 116: 25-34.

15. Frame MJ, Mottram JC, Coombs GH, 2000. Analysis of the roles of cysteine proteinases of Leishmania mexicana in the hostparasite interaction. Parasitology 121: 367-377. 